astronomical factors are of prime importance, but the way in which world-wide climatic changes occur are very much more complex than indicated by Calder.

Such considerations are not merely of academic interest-they become vital as large-scale technology begins to alter the environment. What is the likely climatic effect of diverting Soviet Arctic rivers southwards for irrigation projects? What would be the effect of a permanent ice-free channel for the movement of oil from Arctic Canada? What is the climatic effect of pollutants in the stratosphere? These questions will not be answered until a better dating system for the Quaternary enables the various cycles to be defined. Then the statistical validity of the many possible correlations can be tested and their causative relationship evaluated.

D. H. TARLING

\section{Plants with talent for breeding or competing}

from Peter D. Moore

Population ecologists have made considerable use of the concept of $r$ and $K$ selection, which was introduced by MacArthur and Wilson (The Theory of Island Biogeography, Princeton University Press; 1967). When intraspecific competition (and, therefore, densitydependent mortality) is low, natural selection operates chiefly through its density-independent component, $r$ selection. Taxa associated with such situations are said to be $r$ selected. Conversely, when intraspecific competition and density-dependent mortality are high, $K$ selection is operative. Theoretically, $r$ selection should favour organisms which can breed rapidly, and $K$ selection those which can most efficiently exploit a resource. Pianka ( $\mathrm{Am}$. Nat., 104, 592; 1970 and 106, 581; 1972) has pointed out that no organism is completely $r$ selected or $K$ selected, but lies at some point along a continuum between these extremes.

Investigations into selective pressures in populations of organisms have mainly concentrated upon animals, but Gadgil and Solbrig (Am. Nat., 106, 14; 1972) suggested that selective pressures took a similar form in populations of dandelions (Taraxacum officinale) in different types of grassland at Ann Arbor. Michigan. Since this species is extremely plastic in its morphology it was necessary to use isozyme patterns in leaf extracts to determine genetic biotypes. Four such variants were found, one (A) being associated with the most highly disturbed situations, another (D) being found in stable, undisturbed situations and the other two being intermediate. The series seemed to show a trend from $r$ to $k$ selection.
Solbrig and Simpson (J. Ecol., 62, 473 ; 1974) have now published some experimental data which support this suggestion. Taxa which have been subjected to $r$ selection would be expected to expend a larger proportion of their energy resources in reproductive effort in order to exploit rapidly an unsaturated (low competition) environment. Energy expended per seed, however, need not be high because of the lack of intraspecific competition. Biotype A was found to produce an aver age of 25.3 seeds per plant with a mean seed weight of $0.32 \mathrm{mg}$; biotype $\mathrm{D}$ bore 8.2 seeds per plant with a mean seed weight of $0.44 \mathrm{mg}$. These extreme biotypes thus fulfil the theoretical requirements of $r$ and $K$ strategists in this respect. One would further expect a $K$ selected taxon to be a more effective competitor under high density conditions than an $r$-selected one. This was tested by growing the two biotypes both alone and in combination. When in pure culture there was no significant difference between the biotypes in terms of dry weight increase over 9 ? weeks. When grown together, however, type $\mathrm{D}$ always produced the heavier plants and had the lower mortality rate; it can therefore be regarded as the stronger competitor of the two.

These two extreme biotypes thus demonstrate adaptation apparently in response to differing pressures of $r$ and $K$ selection in their two sites. It is not possible, however, on the basis of these experiments, to determine whether these forms of selection are the most influential ones at work upon the dandelion genomes. Habitats in which $r$ selection is operative are frequently subjected to severe edaphic and microclimatic stresses which could have an overriding selective influence on survival. $K$-selection situations on the other hand experience high levels of interspecific as well as intraspecific competition, which could call for rather different adaptive mechanisms. The apomictic nature and therefore the genetic homogeneity of dandelion biotvpes will make them ideal material for the investigation of these further problems.

\section{Meteor rates, volcanoes and the solar cycle}

\section{from David $W$. Hughes}

A WORLD-WIDE increase in radar meteor echo rates occurred in 1963 and the cause of this has been puzzling scientists ever since. These echoes are obtained by reflecting short $(100 \mu \mathrm{s})$ pulses of radiation (in the tens of $\mathrm{MHz}$ region) from the ionised trains produced in the upper atmosphere (at heights of 80 to $100 \mathrm{~km}$ ) by incoming meteoroid particles. The number of echoes seen in 1963 was a factor of 1.5 to 2 greater than in previous and subsequent years and this increase was recorded in Christchurch, New Zealand ${ }^{1}$, Ottawa, Canada $^{2}$ and Onsala, Sweden ${ }^{3}$, so instrumental effeots can be ruled out. The effect was periodic, in New Zealand occurring during the winter (June, July) and recurring on successive years with reduced magnitude. In the Northern Hemisphere the effect occurred initially in May 1963 but on following years recurred in the winter months, JanuaryMarch. The Swedish data showed a rate increase for all echo duration. Canadian data however indicated that the anomalous increase was restricted to the smaller meteors. The rates were also proportionally higher throughout the day 4

Kennewell and Ellyett of Newcastle University, New South Wales have reconsidered the possible causes of the rate increase in a recent article in Science (186, 355; 1974). They divide the possible causative agents into extraterrestrial and terrestrial.

Consider the extraterrestrial possibility first. There are no reasons why the influx of meteoroid dust to the Earth should not vary from year to year. For example certain meteor showers are distinctly periodic in their activity, the Leonids and Giacobinids being perfect examples. Showers are fed by the decay of the parent comet and it takes a considerable time for the new meteoroids to move around the comet orbit and form a complete, uniformly dense toroid of dust in space. The loop formation times of the Quadrantid and Perseid meteor streams are for example 330 and 4,000 years respectively. The activity of streams which are young and not completely formed is expected to vary with the periodicity of the original comet (33 and 6 years respectively for the Leonids and Giacobinids) the dust still being closely grouped around the comet. A considerable proportion of the sporadic meteoroid background $(\sim 30 \%)$ is made up of minor streams and therefore the mean periodicity of these streams might be reflected in the total influx rate. But the 1963 increase extended over a period of some months which is incompatible with the period of activity of most meteor showers, usually less than a month. Also the fact that the recurring annual variations after 1963 were 6 months out of phase in opposite hemispheres cannot be explained. And the form of the monthly mean diurnal variation remained unchanged-this being something which would vary decidedly if a shower was active. Kennewell and Ellyett conclude that the rate in crease in 1963 was not 